\title{
Functional role of aspartic proteinase cathepsin $D$ in insect metamorphosis
}

Zhong Zheng Gui ${ }^{\dagger 1}$, Kwang Sik Lee ${ }^{\dagger 1}$, Bo Yeon Kim ${ }^{1}$, Yong Soo Choi ${ }^{1}$, Ya Dong Wei ${ }^{1}$, Young Moo Choo ${ }^{1}$, Pil Don Kang ${ }^{2}$, Hyung Joo Yoon ${ }^{2}$, Iksoo Kim³, Yeon Ho Je ${ }^{4}$, Sook Jae Seo ${ }^{5}$, Sang Mong Lee ${ }^{6}$, Xijie Guor, Hung Dae Sohn ${ }^{1}$ and Byung Rae Jin*1

\author{
Address: ${ }^{1}$ College of Natural Resources and Life Science, Dong-A University, Busan 604-714, Korea, ${ }^{2}$ Department of Agricultural Biology, National \\ Institute of Agricultural Science and Technology, RDA, Suwon, Korea, ${ }^{3}$ Department of Agricultural Biology, Chonnam National University, \\ Gwangju, Korea, ${ }^{4}$ School of Agricultural Biotechnology, Seoul National University, Seoul, Korea, ${ }^{5}$ Division of Applied Life Science, Gyeongsang \\ National University, Jinju, Korea, ${ }^{6}$ Department of Life Science and Environmental Chemistry, Pusan National University, Miryang, Korea and \\ ${ }^{7}$ Sericultural Research Institute, Chinese Academy of Agricultural Sciences, Zhenjiang, China \\ Email: Zhong Zheng Gui - srizzgui@hotmail.com; Kwang Sik Lee - nina-k@hanmail.net; Bo Yeon Kim - 79ehtnr@hanmail.net; \\ Yong Soo Choi - bioboy@korea.com; Ya Dong Wei - yadongww@yahoo.com; Young Moo Choo - youngmoo76@hanmail.net; \\ Pil Don Kang - kangpd@rda.go.kr; Hyung Joo Yoon - yoonhj@rda.go.kr; Iksoo Kim - ikkim81@chonnam.ac.kr; Yeon Ho Je - btrus@snu.ac.kr; \\ Sook Jae Seo - sookjae@gshp.gsnu.ac.kr; Sang Mong Lee - serilsm@hanmail.net; Xijie Guo - guoxijie@hotmail.com; \\ Hung Dae Sohn - hdsohn@dau.ac.kr; Byung Rae Jin* - brjin@dau.ac.kr \\ * Corresponding author †Equal contributors
}

Published: 25 October 2006

BMC Developmental Biology 2006, 6:49 doi:10.1186/147/-213X-6-49

This article is available from: http://www.biomedcentral.com/I47I-2/3X/6/49

(C) 2006 Gui et al; licensee BioMed Central Ltd.

This is an Open Access article distributed under the terms of the Creative Commons Attribution License (http://creativecommons.org/licenses/by/2.0), which permits unrestricted use, distribution, and reproduction in any medium, provided the original work is properly cited.

\begin{abstract}
Background: Metamorphosis is a complex, highly conserved and strictly regulated development process that involves the programmed cell death of obsolete larval organs. Here we show a novel functional role for the aspartic proteinase cathepsin $D$ during insect metamorphosis.

Results: Cathepsin D of the silkworm Bombyx mori (BmCatD) was ecdysone-induced, differentially and spatially expressed in the larval fat body of the final instar and in the larval gut of pupal stage, and its expression led to programmed cell death. Furthermore, BmCatD was highly induced in the fat body of baculovirus-infected $B$. mori larvae, suggesting that this gene is involved in the induction of metamorphosis of host insects infected with baculovirus. RNA interference (RNAi)-mediated $B m C a t D$ knock-down inhibited programmed cell death of the larval fat body, resulting in the arrest of larval-pupal transformation. BmCatD RNAi also inhibited the programmed cell death of larval gut during pupal stage.
\end{abstract}

Conclusion: Based on these results, we concluded that BmCatD is critically involved in the programmed cell death of the larval fat body and larval gut in silkworm metamorphosis.

\section{Background}

Insect metamorphosis is a complex, highly conserved, and strictly regulated process of developmental events. Metamorphosis is triggered by the steroid hormone ecdysone in the absence of the sesquiterpenoid juvenile hormone and is carried out by self-destructive mechanisms of programmed cell death [1]. The developmental process of different larval tissues during metamorphic transformation 
showed that tissues such as the silk gland and gut are completely histolyzed [2-4], while other tissues such as fat body undergo reorganization with histolysis $[5,6]$, and predetermined imaginal tissues differentiate and grow into organs and external structures $[4,7]$.

The ecdysone-induced transcription factor Broad-Complex (BR-C) plays an important regulatory role in metamorphosis [8-14]. It is required for differentiation of adult structures as well as for the programmed death of obsolete larval organs during metamorphosis. The Bombyx BR-C RNAi disrupted the differentiation of adult compound eyes, legs and wings, and also perturbed the programmed cell death of larval silk glands [4].

Additionally, the Bombyx BR-C function uncovers the programmed cell death of larval fat body and larval gut during silkworm metamorphosis. It is still unclear what gene products function in the programmed cell death of larval fat body and/or larval gut. Therefore, we asked whether cathepsins are involved in the metamorphic events of silkworm because, to date, studies in insects reveal that cathepsins also participate in developmental processes [2,1523]. Recently, a study has shown that the temporal activity profile of an aspartic proteinase is associated with fat body histolysis during Ceratitis capitata early metamorphosis [6]. Studies of insect cathepsins strongly implicate the involvement of activated proteinases in metamorphic events. Thus, it is of interest to know whether cathepsin has any functional roles in insect metamorphosis through a loss-of-function test.

Here, we have focused on cathepsin D, a lysosomal aspartic proteinase, as a metamorphosis-specific proteinase involved in metamorphic events. To help elucidate the molecular mechanisms of metamorphosis in the silkworm, we first cloned the Bombyx mori cathepsin D (BmCatD) gene from the silkworm. We examined the expression profile of $B m C a t D$ during development; $B m C a t D$ is induced by the steroid hormone ecdysone and baculovirus infection, and is expressed in a tissue- and developmental stage-specific pattern in the larval fat body of the final instar and in the larval gut of pupal stage. Finally, we demonstrate that loss of BmCatD function disrupts two classes of metamorphic events in Bombyx, larvalpupal transformation and programmed cell death of larval gut.

\section{Results and discussion}

\section{A novel aspartic proteinase (BmCatD) gene cloned from} the silkworm B. mori

The BmCatD CDNA was isolated by searching B. mori ESTs that encode a protein of 385 amino acids (GenBank accession number AY297160). Comparison of amplicon size between the genomic and cDNA sequences revealed the presence of nine exons and eight introns in BmCatD (Fig. 1A). The two catalytic centers and aspartic acid residues, as well as the six cysteine residues characteristic of aspartic proteinases $[15,24,25]$, were conserved in $\mathrm{BmCatD}$, indicating that BmCatD is a member of the same family as all other insect aspartic proteinases identified to date. BmCatD showed the closest amino acid identity with the aspartic proteinase of the mosquitoes Anopheles gambiae (64\% identity) and Aedes aegypti (63\% identity). However, this BmCatD gene did not align with any lepidopteran CatD gene identified to date.

Cathepsin D has been reported to be an N-glycosylated high mannose glycoprotein that functions as an acidic proteinase, with an optimal pH of $3.0[6,15,26]$. We found that recombinant BmCatD expressed in baculovirus-infected insect cells was N-linked glycosylated, but its $\mathrm{N}$-linked glycosylation is not necessary for enzyme activity and that the purified recombinant BmCatD exhibited a high proteolytic activity at $\mathrm{pH}$ 3.0-3.5, establishing $\mathrm{BmCatD}$ as an aspartic proteinase (Fig. 1B,c).

\section{BmCat $D$ is expressed in a developmental stage- and tissue- specific manner and its expression causes programmed cell death}

To examine the expression of BmCatD in various tissues during development, we first performed Northern blot analysis. We found that $B m C a t D$ was expressed in the fat body of the final (fifth) larval instar, but not in the pupal fat body, and was also seen in the gut during pupal development. Next, developmental stage- and tissue-specific profiles of BmCatD expression were performed using total RNA obtained from fat body and gut during the fifth larval instar to pupal stages (Fig. 2A). In the fat body, $B m C a t D$ mRNA was expressed from day 3 in the fifth larval instar to day 2 in the spinning stage (upper panel of Fig. 2B). On the other hand, BmCatD mRNA in larval gut was expressed during the entire pupal stage including prepupae (upper panel of Fig. 2C). The expression level of $B m C a t D$ was relatively low in the prepupal stage, increased dramatically on day 1 of the pupal stage, and thereafter gradually reduced but remained until adult eclosion. These results showed that the expression pattern of $B m C a t D$ was specifically started in the fat body on day 3 of the fifth larval instar and completely disappeared on day 1 of the prepupal stage. In larval gut, expression of $B m C a t D$ was precisely detected on day 1 of the prepupal stage, indicating that $B m C a t D$ is differentially and spatially expressed during development and its expression proceeds in a precise tissue- and developmental stagedependent manner. Consistent with Northern blot data, the expression pattern of BmCatD protein, which was confirmed by Western blotting using the antibody of recombinant BmCatD that was expressed in baculovirusinfected insect cells, was observed in the fat body of the 


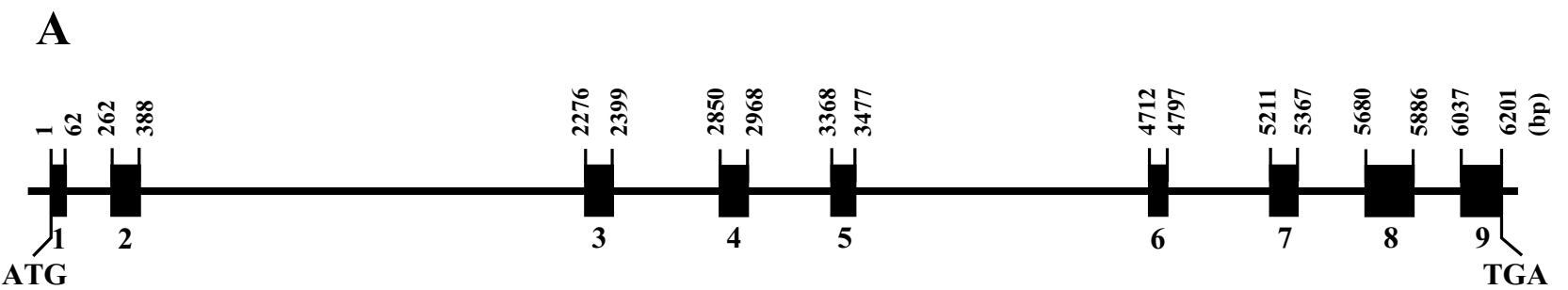

B

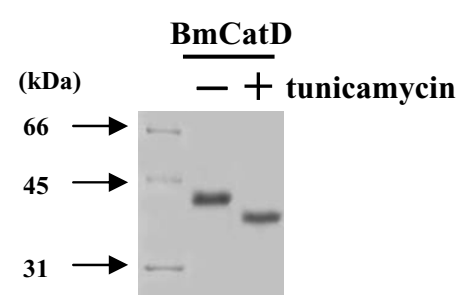

C

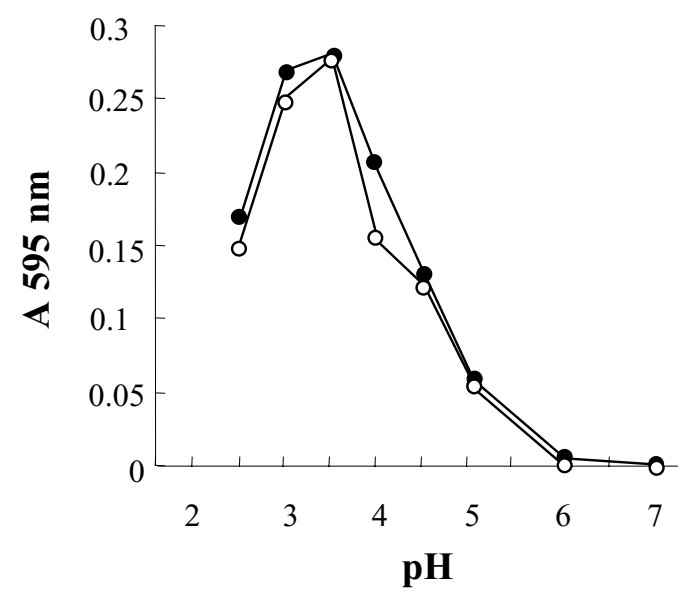

\section{Figure I}

Characterization of $B m C a t D$ gene and protein product. $(A)$ Genomic structure of $B m C a t D$ gene revealed by PCR amplification from $B m C a t D$ cDNA. Numbers indicate the position in the genomic sequences. GenBank accession numbers are AY297I60 (BmCatD cDNA) and DQ4I7605 (BmCatD genomic DNA). (B) N-linked glycosylation of recombinant BmCatD expressed in baculovirus-infected insect Sf9 cells. The recombinant AcNPV-infected Sf9 cells were treated with (+) or without (-) tunicamycin and the cell lysates were analyzed by Western blot analysis. (C) Optimum pH of recombinant BmCatD. The N-linked glycosylated (solid circle) and nonglycosylated (open circle) BmCatD polypeptides were purified from culture supernatants. The $\mathrm{pH}$ dependency of recombinant BmCatD activity on $2 \%$ hemoglobin was assayed directly at different $\mathrm{pHs}$.

fifth larval instar (middle panel of Fig. 2B) and in the larval gut of pupal stage (middle panel of Fig. 2C).

The prospective fates of these tissues such as fat body and gut during metamorphic transformation are different. The larval gut is completely histolyzed during the pupal stage [2-4]; on the other hand, the fat body undergoes reorganization with histolysis during larval-pupal transformation $[5,6]$. It seems logical that the histolysis of larval fat body and larval gut represents a programmed cell death response. Therefore, we assayed whether the histolysis of larval fat body and larval gut is accompanied by internucleosomal DNA fragmentation that is a rapid and accurate indicator of the involvement of apoptosis in cell death $[6,9]$. To determine whether the BmCatD expression correlates with the histolysis of larval fat body and larval gut during metamorphosis, we analyzed the induction of pro- grammed cell death in the fat body and gut tissues. Figure 2 also shows internucleosomal DNA fragments, seen as programmed cell death-specific laddering on agarose gel electrophoresis, for the fat body and gut during metamorphosis. The DNA fragmentation by histolysis of larval fat body was observed from day 3, peaked on day 7 in the fifth instar, and dramatically reduced on day 2 in the spinning stage (lower panel of Fig. 2B). In the larval gut, DNA fragmentation initiated on day 1 of the pupal stage and thereafter gradually increased until adult eclosion (lower panel of Fig. 2C). These results suggest that in fat body and gut, BmCatD expression was accompanied with DNA fragmentation. In addition, the DNA fragmentation rapidly and severely occurred in larval gut in the pupal stage. The result suggests that the developmental profiles of BmCatD expression, as judged by DNA fragmentation, differed between the larval gut, which undergoes programmed cell 

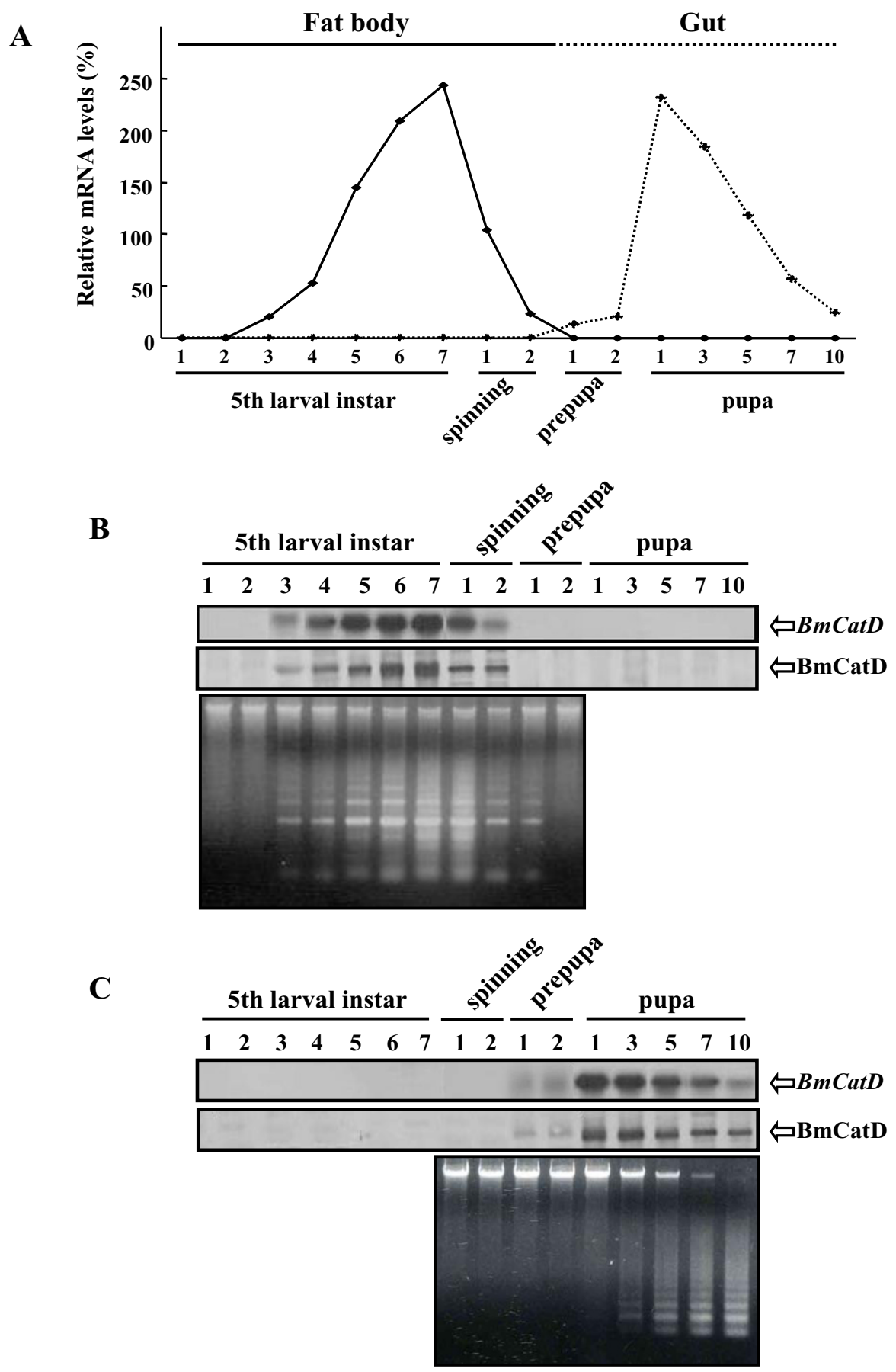

\section{Figure 2}

BmCatD expression profile and internucleosomal DNA fragmentation in the fat body and gut of $B$. mori. (A) Relative mRNA levels of $B m C a t D$ during the fifth larval instar to pupal stage. The level of BmCatD mRNA is calculated relative to that of the background (shown as $0 \%$ ). The fat body and gut of $B$. mori were collected during the fifth larval instar to pupal stage (as indicated), respectively. $(B, C) B m C a t D$ expression and internucleosomal DNA fragmentation in the fat body (B) and gut (C) of $B$. mori. The expression level of $B m C a t D$ mRNA (upper) and its protein (middle) was analyzed by Northern blot and Western blot analyses, respectively. Internucleosomal DNA fragmentation in fat body and gut cells was assessed by $1.0 \%$ agarose gel electrophoresis (lower). 
death during the pupal stage, and larval fat body, which survives into the adult phase but undergoes reorganization during larval-pupal transformation.

\section{$B m C a t D$ is induced by ecdysone and viral infection}

Metamorphosis is regulated by changes in the titer of the steroid hormone ecdysone in the absence of the sesquiterpenoid juvenile hormone $[1,27,28]$. In order to determine if $B m C a t D$ expression is an ecdysone-triggered response, we examined effects of 20-hydroxyecdysone (20E) and juvenile hormone analogue (JHA) on BmCatD mRNA expression using the fat body of the fifth instar larvae. When 20E was injected into day 1 fifth instar larvae, $B m C a t D$ mRNA was clearly detected in the fat body on day 2 of the fifth larval instar (Fig. 3A-b), although no BmCatD expression was observed in the control during this period (Fig. 3A-a). On the other hand, topical application of JHA reduced BmCatD expression (Fig. 3A-c). These results show that $B m C a t D$ expression is up-regulated by $20 \mathrm{E}$ and down-regulated by JHA (Fig. 3B), implicating that these hormonal responses can explain ecdysone-induced expression of BmCatD in the fifth larval instar. Especially, 20E administration highly induced programmed cell death of larval fat body (Fig. 3C), indicating that the onset of larval fat body histolysis coincides with the expression of BmCatD. During insect metamorphosis, pulses of the steroid hormone ecdysone direct the destruction of obsolete larval tissues and the replacement of larval tissues and structures with adult forms [9]. Day 3 of the fifth larval instar in the silkworm is a critical period for larval growth, and during this period, prothoracic glands begin to secrete detectable ecdysteroid $[27,28]$. A pulse of ecdysone at the end of larval development triggers pupation, and the ecdysteroid activity induced by another ecdysone pulse again increases dramatically just before pupation $[27,28]$. The larval silk gland and larval gut begin programmed cell death in response to an increase in ecdysteroid concentration in the pupal stage [29-31]. Taken together, our results indicate that $B m C a t D$ is expressed exclusively in the larval fat body on day 3 of the fifth instar and in the larval gut on day 1 of the prepupal stage, and suggest that the expression of $B m C a t D$ is regulated in a tissue- and developmental stage-specific manner by ecdysone fluctuations.

Host insect infection with baculovirus induces metamorphosis $[32,33]$. This raises the possibility that BmCatD may be involved in the induction of metamorphosis of host insects infected with baculovirus. To examine this possibility, we carried out transcriptional induction analysis of BmCatD in baculovirus-infected silkworm larvae. When BmNPV was injected into larvae on day 1 of the fifth instar, BmCatD mRNA was detected in the fat body on day 2 of the fifth larval instar (Fig. 3A-d), although no BmCatD expression was observed in the controls (Fig. 3Aa). Consistent with this, the induced programmed cell death of larval fat body by viral infection was clearly observed on day 2 of the fifth larval instar (Fig. 3C). Furthermore, the level of $B m C a t D$ expression was found to be higher in virus-infected larvae than in uninfected controls (Fig. 3B). This result indicates that viral infection induced the BmCatD expression, which resulted in the induced programmed cell death in larval fat body, and suggests that BmCatD was involved in the induction of metamorphosis in the host insect infected with baculovirus. During virus replication, the prothoracic gland of host insects was observed to maintain characteristics indicative of ecdysone biosynthetic activities [33]. The haemolymph ecdysteroid level and prothoracic gland activity in baculovirusinfected larvae were higher than in controls, which continued until the late stages of viral infection, even during the time that controls had ceased to secrete ecdysone after molting $[32,33]$. Thus, these observations indicate that viral infection resulted in the up-regulation of $B m C a t D$, as shown in $20 \mathrm{E}$ treatment, and suggest that BmCatD up-regulation is the result of alteration of host's hormonal system by viral infection.

To further understand the correlation between hormonal system and viral infection, we injected larvae on day 1 of the fifth instar with Bombyx mori nucleopolyhedrovirus (BmNPV) just after JHA treatment, which down-regulates $B m C a t D$ expression. Interestingly, in both JHA and BmNPV treatments, BmCatD expression was up-regulated in a viral infection-dependent manner (Fig. 3A-e), thus indicating that viral infection plays a role in the regulation of BmCatD expression, regardless of the presence or absence of JHA. The host's hormonal system, altered by viral infection, induces metamorphosis [32,33], which suggests that metamorphosis induction due to virus infection functions as a viral defense system of the host insect. In contrast, baculoviruses are known to contain the unique $p 35$ gene, which blocks virus-induced apoptosis [34]. Therefore, the inhibition of DNA fragmentation at later stages of viral infection (Fig. 3C) was likely due to P35 of BmNPV. This result was consistent with previous observations indicating that baculovirus infection blocks the progression of fat body degradation [35].

\section{Loss of BmCatD function disrupts metamorphic events in the silkworm}

It is known that in the fat body of insects, the lysosomal system is involved in cellular remodeling, which is associated with metamorphosis and termination of egg maturation cycles $[6,15,36,37]$. However, there has been relatively little research into the substantial functional role of CatD in metamorphosis in insects, especially in Lepidoptera. Therefore, we next asked whether BmCatD is functionally involved in the metamorphosis of the silkworm, and whether silkworms have any defects in the $B m C a t D$ RNA interference (RNAi) process. In order to 

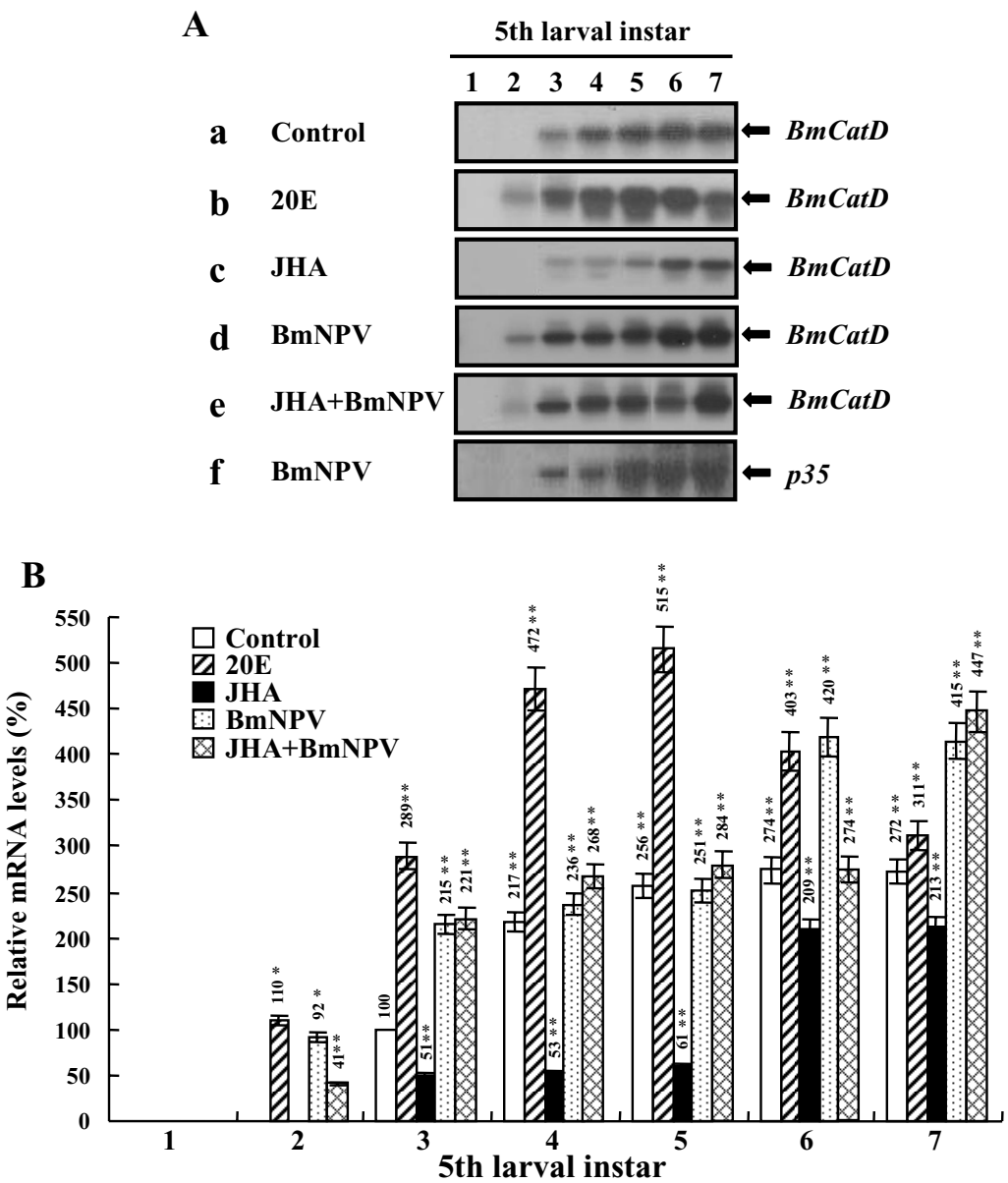

C

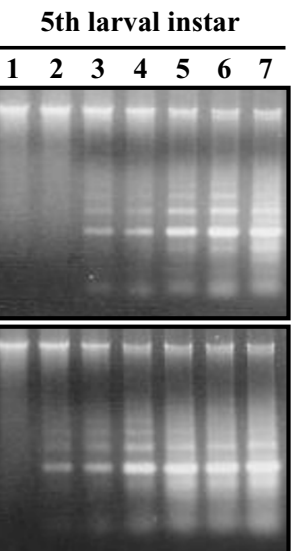

JHA

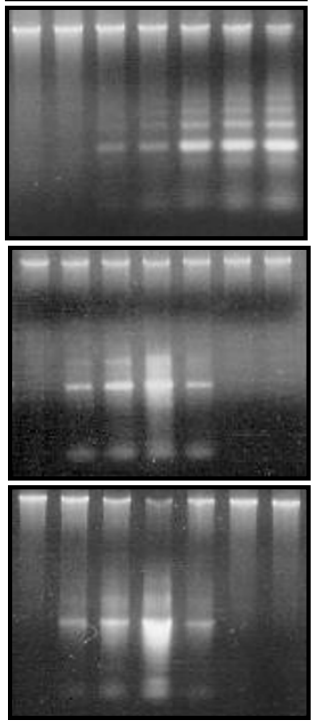

\section{Figure 3}

Hormonal and viral regulation of $B m C a t D$ mRNA expression. (A) Expression profiles of $B m C a t D$ mRNA in fat body of the fifth larval instar with hormonal and viral treatments. B. mori larvae on day I of the fifth instar were treated with $20 \mathrm{E}$ (b), JHA (c), BmNPV (d) or JHA+BmNPV (e), respectively. Total RNA from fat body was extracted at I-day intervals post-treatment and analyzed by Northern blots. The control was the untreated larvae (a). BmNPV p35 ( $f$ ) gene serves as a marker of viral infection. (B) Relative mRNA levels of BmCatD regulated by treatment. The levels of BmCatD mRNA are means of three assays, which are calculated relative to that of the expression recorded for the control (shown as $100 \%$ ). Bars represent the means \pm SE. Significance of the differences versus a control value is given by $* *(P<0.01)$ and $*(P<0.05)$. (C) Internucleosomal DNA fragmentation in the fat body of the fifth larval instar with hormonal and viral treatments. DNA from the fat body cells of all treated larvae was extracted at I-day intervals post-treatment (as described in A), respectively. DNA fragmentation was assessed by $1.0 \%$ agarose gel electrophoresis.

determine the functional role of BmCatD in silkworms during metamorphosis, we reduced the endogenous BmCatD mRNA levels in the fat body of silkworm larvae by using RNAi, and then confirmed the reduced BmCatD mRNA levels by using Northern blot hybridization. $B m C a t D$ mRNA levels in fat body of BmCatD RNAi-mediated silkworm larvae were significantly reduced compared to the control silkworm larvae (Fig. 4A). To characterize the functional role of BmCatD in silkworm larvae, it is important to obtain silkworm larvae containing little or no BmCatD. This approach relies on the degree of $B m C a t D$ reduction caused by RNAi, as a measure of RNAi function, in order to explore the proposed role of BmCatD in silkworm larvae.

In BmCatD RNAi-mediated silkworm larvae, larval-pupal metamorphosis was strongly affected by BmCatD RNAi. Compared to controls, all of which underwent regular larval-pupal transformation, three-fourths of BmCatD RNAi on day 3 fifth instar larvae arrested during larval-pupal 
A

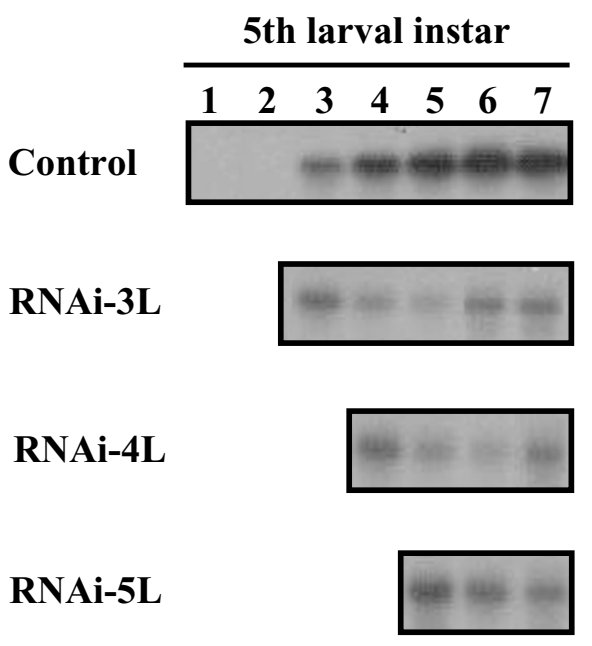

B

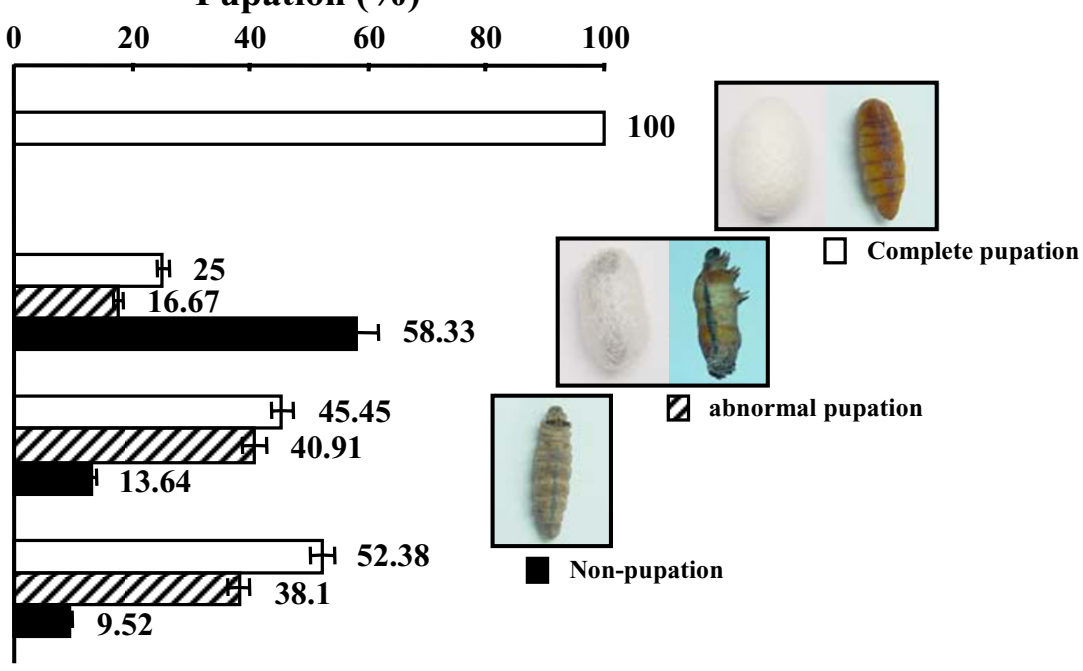

C

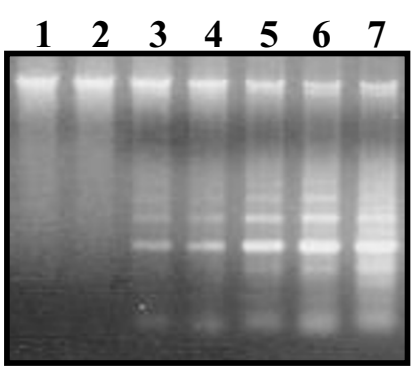

Control

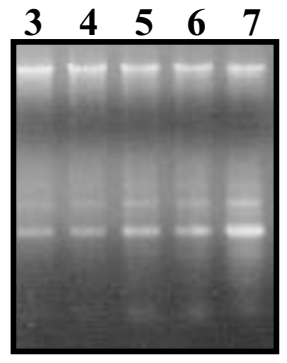

RNAi-3L

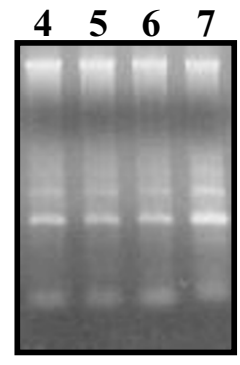

RNAi-4L

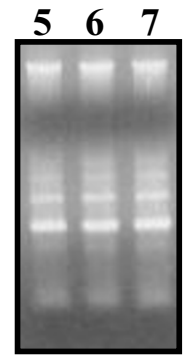

RNAi-5L

\section{Figure 4}

Effects of BmCatD RNAi on B. mori development. (A) BmCatD expression profile in RNAi-mediated B. mori larvae and controls. $B m C a t D$ dsRNA was injected into larvae on day 3 (RNAi-3L), 4 (RNAi-4L) and 5 (RNAi-3L) of the fifth instar, respectively. The control was the untreated larvae. Total RNA from the fat body was extracted at I-day intervals post-treatment. The expression level of BmCatD mRNA was analyzed by Northern blot analysis. (B) Pupation rate in RNAi-mediated B. mori larvae and controls. The pupation rates are the means of three assays. Bars represent the means \pm SE. (C) Internucleosomal DNA fragmentation of the fat body in RNAi-mediated B. mori larvae. DNA from the fat body cells of all treated larvae was extracted at I-day intervals post-treatment (as described in A), respectively. DNA fragmentation was assessed by $1.0 \%$ agarose gel electrophoresis.

metamorphosis (Fig. 4B). In this case, a large portion of BmCatD RNAi-mediated silkworm larvae was nonpupated $(58.3 \%)$ and $16.7 \%$ of larvae were abnormally pupated. Similar effects were respectively seen in 55\% and $48 \%$ of BmCatD RNAi on day 4 and 5 fifth instar larvae, and the abnormal pupation rate was relatively high in these cases (Fig. 4B). The arrest of larval-pupal transformation was observed in BmCatD RNAi-mediated silkworm larvae and indicated that BmCatD is necessary for the larval-pupal metamorphosis in the silkworm. It is for- mally conceivable that BmCatD might have additional functions in other developmental processes, and reduction in BmCatD via RNAi in these processes might directly or indirectly contribute to developmental arrest.

We next tried to determine whether BmCatD could induce DNA laddering in larval gut during pupal development. To provide evidence that BmCatD is involved in programmed cell death of larval gut, we also reduced the endogenous BmCatD mRNA levels in the gut of silkworm 
pupal stage by using RNAi and then examined the pattern of DNA fragmentation in the larval gut. As observed from the Northern blots (lower panels of Fig. 5), BmCatD levels were reduced in the larval gut of $B m C a t D$ RNAi-mediated silkworm pupae. Compared to controls, which undergo rapid and severe DNA fragmentation in the larval gut from day 3 of the pupal stage, BmCatD RNAi-mediated silkworm pupae exhibited an inhibition of DNA fragmentation in larval gut (upper panels of Fig. 5). When BmCatD dsRNA was injected repeatedly into pupae on day 1 and 5 of the pupal stage, the inhibition of DNA fragmentation in larval gut was more clearly affected. These results indicate that loss of BmCatD causes a defect in internucleosomal DNA fragmentation of larval gut, pointing to an important role of BmCatD in programmed cell death of larval gut.

As judged from the observed effect of BmCatD RNAi during metamorphosis, all observations in this study provide strong evidence that BmCatD was involved in the histolysis of larval fat body and larval gut, demonstrating a functional involvement as a metamorphosis-specific lysosomal proteinase. Recently, most studies of molecular mechanisms of metamorphosis in silkworm have focused on the metamorphosis-specific transcriptional factor BR-
$\mathrm{C}[4,38-40]$. It has been shown that the Bombyx BR-C is expressed in an ecdysone-induced manner and is required for programmed cell death of larval silk glands, as well as for the differentiation of adult structures including compound eyes, legs, and wings [4]. By focusing our findings on the BmCatD, we have been able to explain metamorphosis-specific functions of BmCatD.

\section{Conclusion}

The work provided here demonstrates the involvement of cathepsin D as a metamorphosis-specific proteinase in metamorphic events. This finding is important in that it sheds new light on the functional role of cathepsin D in silkworm metamorphosis. The metamorphic defects seen in the BmCatD RNAi-mediated silkworm, such as larvalpupal transformation arrest and programmed cell death inhibition, highlight an important functional role of BmCatD in metamorphic processes and provide a foundation for a better understanding of the molecular mechanisms of insect metamorphosis.

\section{Methods}

\section{Experimental animals}

Larvae of the silkworm, Bombyx mori, used in this study were F1 hybrid Baekok-Jam supplied by Department of

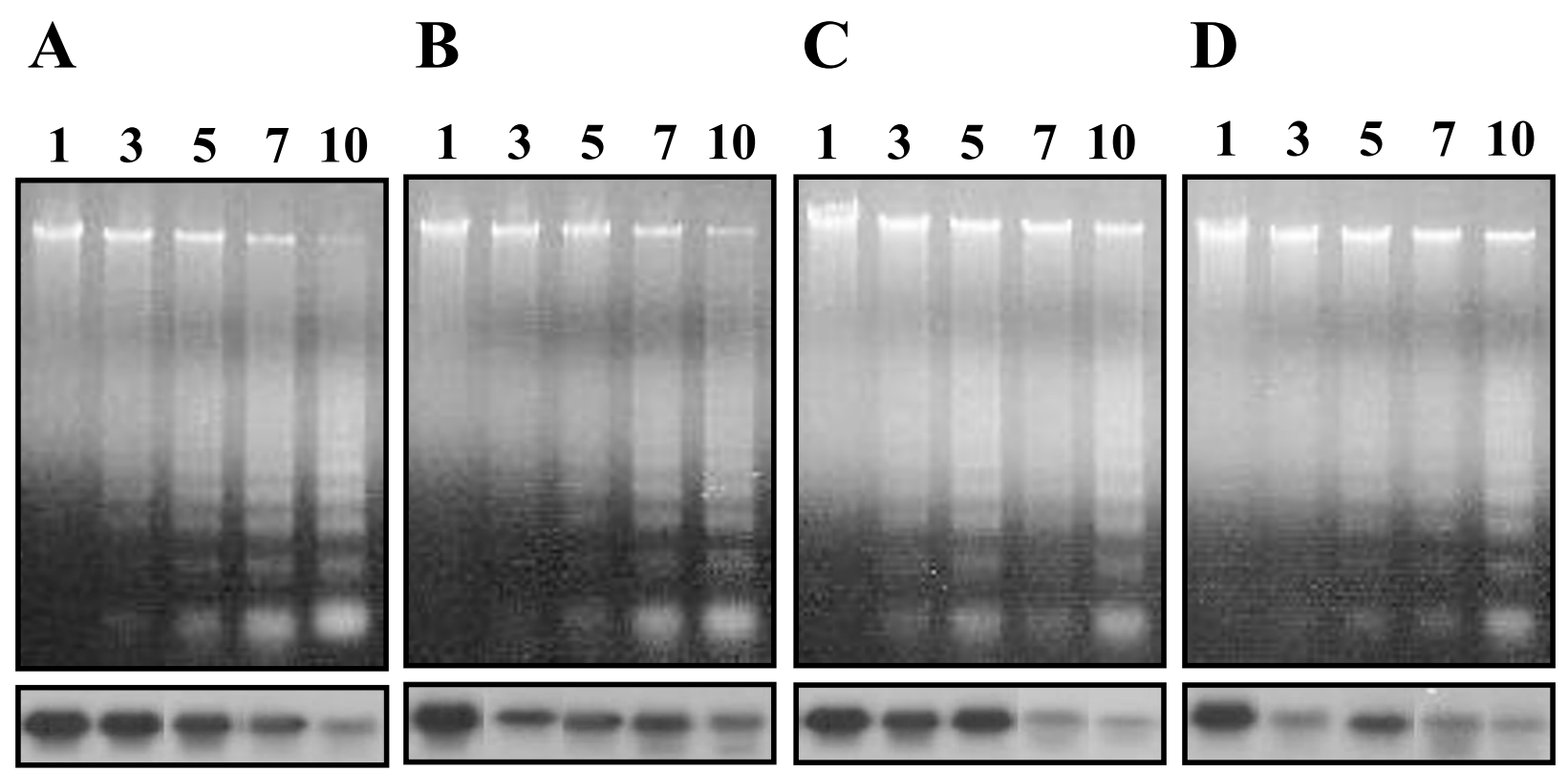

\section{Figure 5}

Internucleosomal DNA fragmentation of the larval gut in RNAi-mediated $B$. mori pupae. BmCatD dsRNA was injected into pupae on day I (B), 5 (C) or both I and 5 (D) of the pupal stage, respectively. The control was the untreated pupae (A). DNA was extracted from the gut on day I, 3, 5, 7, and 10 of the pupal stage (as indicated), respectively. DNA fragmentation was assessed by $1.0 \%$ agarose gel electrophoresis (upper). Total RNA from larval gut was extracted as above. The expression level of BmCatD mRNA was analyzed by Northern blot analysis (lower). 
Agricultural Biology, The National Institute of Agricultural Science and Technology, Korea. Silkworms were reared on fresh mulberry leaves at $25^{\circ} \mathrm{C}, 65 \pm \%$ relative humidity, and $12 \mathrm{~h}$ light: $12 \mathrm{~h}$ dark photoperiod. Spinning (wandering) occurred on day 7 of the fifth instar, and pre-pupation and pupation occurred 2 days and 3 days thereafter. The first days corresponding to the developmental stages of the fifth larval ecdysis, spinning, and pupation were designated as day 1 of the fifth larval instar, spinning, and pupal stage, respectively.

\section{Gene cloning}

The BmCatD cDNA was cloned from a cDNA library using whole bodies of $B$. mori larvae [41]. The sequences were compared using the DNASIS and BLAST programs provided by the NCBI [42]. MacVector (ver. 6.5, Oxford Molecular Ltd) was used to align the amino acid sequences of CatD. Genomic DNA, extracted from the fat body of single B. mori larva using a Wizard Genomic DNA Purification Kit (Promega), was used for PCR amplification with oligonucleotide primers designed from the $B m C a t D$ cDNA sequences. The nucleotide sequence was determined as described previously [41].

\section{Protein analysis}

A baculovirus expression vector system [43], using the Autographa californica nucleopolyhedrovirus (AcNPV) and an insect cell line Sf9, was employed for the production of recombinant BmCatD protein. Recombinant BmCatD purification, antibody preparation, and Western blot analysis were performed as described previously [44]. The loading volume of protein samples in all Western blot analyses was $5 \mu \mathrm{g} /$ lane. Tunicamycin treatment was performed as described previously [45]. Aspartic proteinase activity of BmCatD was measured as described previously [6].

\section{RNA analysis}

Total RNA was isolated as described [44]. Northern blot and its image analysis were performed as described previously [44]. The loading volume of total RNA in all Northern blot analyses was $5 \mu \mathrm{g} / \mathrm{lane}$.

\section{DNA fragmentation assay}

DNA fragmentation from larval fat body and larval gut was assayed using an Apoptotic DNA-Ladder Kit (Roche Applied Science, Germany) according to the manufacturer's protocols. DNA was analyzed on a $1.0 \%$ agarose gel and visualized by ethidium bromide staining.

\section{Hormonal treatment and viral injection}

Twenty-hydroxyecdysone (20E, Sigma) was dissolved in distilled water and stored at $-20^{\circ} \mathrm{C}$ until used. Twenty micrograms of $20 \mathrm{E}$ dissolved in $20 \mu \mathrm{l}$ of distilled water was injected into B. mori larvae on day 1 of the fifth instar.
Fifty nanograms of a juvenile hormone analogue, fenoxycarb (Sankyo, Japan), dissolved in $20 \mu$ l of acetone were applied topically to larvae with a micropipette along the dorsal midline. For viral infection, BmNPV $[44,46]$ was suspended in TC100 medium. B. mori larvae on day 1 of the fifth instar were injected with $50 \mu \mathrm{l}$ of a viral suspension $\left(1.0 \times 10^{5} \mathrm{PFU} /\right.$ larva $)$. The fat body from all treated larvae was collected at 1-day intervals post-treatment and washed twice with PBS. Total RNA and genomic DNA were isolated from the tissues as described above. For injection in experiments, larvae of $B$. mori were injected with a sample solution between the first and second abdominal segments with a microsyringe. Each assay was replicated three times based on three independent tissue preparations. For comparison of relative BmCatD mRNA levels, statistical analysis of images of Northern blots was performed with Tukey's pairwise comparison test. Results are shown as mean \pm SE of three animals per group. Significant $P$ values were obtained by Tukey's test.

\section{RNAi}

The MEGAscript RNAi kit (Ambion) was used to generate double-stranded RNA (dsRNA) corresponding to nucleotides 226 to 741 of the BmCatD cDNA. T7 promoter sites were added to the PCR primers BmCatD-Fi (5'GCGTAATACGACTCACTATAGGGAGACCGCAGTCGTTCAAGGTGGTA-3') and BmCatD-Ri (5'GCGTAATACGACTCACTATAGGGAGAGAACTCCCAGTACGTGTCCCG-3'). PCR reactions were conducted to generate complementary templates with a single $\mathrm{T} 7$ promoter site. T7 RNA polymerase was used to transcribe single-stranded RNA (ssRNA) from each DNA template over $4 \mathrm{~h}$ at $37^{\circ} \mathrm{C}$. BmCatD dsRNA was produced by mixing solutions containing equal amounts of complementary ssRNA, incubating at $75^{\circ} \mathrm{C}$ for $5 \mathrm{~min}$, and allowing the solution to cool down to room temperature. DNA and ssRNA were removed from the solution by digestion with DNase I and RNase at $37^{\circ} \mathrm{C}$ for $1 \mathrm{~h}$. The dsRNA was purified using purification cartridges provided in the kit and dsRNA was eluted with two successive $50 \mu \mathrm{l}$ washings of pre-heated $\left(95^{\circ} \mathrm{C}\right) 10 \mathrm{mM}$ Tris- $\mathrm{HCl}(\mathrm{pH} 7.0)$ containing 1 mM EDTA. Finally, total dsRNA was quantified from the $\mathrm{A}_{260}$. BmCatD dsRNA $\left(\approx 1 \mathrm{mg} \mathrm{ml}^{-1}\right)$ was injected into larvae or pupae of $B$. mori (injection volume $\approx 50 \mu \mathrm{l} /$ individual) using a sterile needle. After injection, all individuals were covered with paraffin.

\section{Authors' contributions}

ZZG and KSL carried out most of the experiments in this study. BYK and YDW participated in the RNAi and DNA fragmentation assays. YSC and YMC assisted with cell culture, protein purification and antibody production. PDK carried out silkworm rearing. HJY and HDS assisted with the physiological characterization of the silkworm. IK helped to draft the manuscript. YHJ helped with the bac- 
ulovirus expression vector and protein expression. SJS, SML, and XG assisted with the analysis of results. BRJ was responsible for the experiment design, analysis and interpretation of data and writing of manuscript. All authors approved the final manuscript.

\section{Acknowledgements}

This work was supported by a grant from the Biogreen 2I Program, Rural Development Administration, Republic of Korea and the Brain Korea 21 Project, the Ministry of Education, Republic of Korea.

\section{References}

I. Riddiford LM: Hormone action at the cellular level. In Comprehensive Insect Physiology, Biochemistry, and Pharmacology Edited by: Kerkut GA, Gilbert LI. New York: Pergamon Press; 1985:37-84.

2. Shiba $H$, Uchida D, Kobayashi $H$, Natori M: Involvement of cathepsin B- and L-like proteinases in silk gland histolysis during metamorphosis of Bombyx mori. Arch Biochem Biophys 2001, 390:28-34.

3. Lee CY, Cooksey BAK, Baehrecke EH: Steroid regulation of midgut cell death during Drosophila development. Dev Biol 2002 250: $101-111$.

4. Uhlirova M, Foy BD, Beaty BJ, Olson KE, Riddiford LM, Jindra M: Use of Sindbis virus-mediated RNA interference to demonstrate a conserved role of Broad-Complex in insect meramorphosis. Proc Natl Acad Sci USA 2003, 100:15607-I56/2.

5. Rizki TM: Fat body. In The Genetics and Biology of Drosophila Edited by: Ashburner M, Wright TFR. New York: Academic Press |978:561-60l.

6. Rabossi A, Stoka V, Puizdar V, Turk V, Quesada-Allué LA: Novel aspartyl proteinase associated to fat body histolysis during Ceratitis capitata early metamorphosis. Arch Insect Biochem Physiol 2004, 57:51-67.

7. Fristrom $D$, Chihara $C$ : The mechanism of evagination of imaginal disc of Drosophila melanogaster : V. Evagination of disc fragments. Dev Biol 1978, 66:564-570.

8. Bayer CA, von Kalm L, Fristrom JW: Relationships between protein isoforms and genetic functions demonstrate functiona redundancy at the Broad-Complex during Drosophila metamorphosis. Dev Biol 1997, 187:267-282.

9. Jiang C, Baehrecke EH, Thummel CS: Steroid regulated programmed cell death during Drosophila metamorphosis. Development 1997, I 24:4673-4683.

10. Jiang C, Lamblin AFJ, Steller H, Thummel CS: A steroid-triggered transcriptional hierarchy controls salivary gland cell death during Drosophila metamorphosis. Mol Cell 2000, 5:445-455.

II. Kiss I, Beaton AH, Tardiff J, Fristrom D, Fristrom JW: Interactions and developmental effects of mutations in the Broad-Complex of Drosophila melanogaster. Genetics 1988, I I 8:247-259.

12. Zhou BH, Hiruma K, Shinoda T, Riddiford LM: Juvenile hormone prevents ecdysteroid-induced expression of broad complex RNAs in the epidermis of the tobacco hornworm, Manduca sexta. Dev Biol 1998, 203:233-244.

13. Brennan CA, Li TR, Bender M, Hsiung F, Moses K: Broad-complex, but not Ecdysone receptor, is required for progression of the morphogenetic furrow in the Drosophila eye. Development 2001, I 28: I- II.

14. Zhou BH, Riddiford LM: Hormonal regulation and patterning of the Broad-Complex in the epidermis and wing disc of the tobacco hornworm, Manduca sexta. Dev Biol 200I, 231:125-137.

15. Cho WL, Raikhel AS: Cloning of cDNA for mosquito lysosomal aspartic protease. J Biol Chem 1992, 267:21823-21829.

16. Kurata S, Saito H, Natori S: The $\mathbf{2 9} \mathbf{~ K d a}$ hemocyte proteinase in dissociated fat body at metamorphosis of Sarcophaga. Dev Biol 1992, 153:115-121.

17. Homma K, Kurata S, Natori S: Purification, characterization, and cDNA cloning of procathepsin $L$ from the cultyre medium of NIH-Sape-4, an embryonic cell line of Sarcophaga peregrina (flesh fly), and its involvement in the differentiation of imaginal disc. J Biol Chem 1994, 269:| 5258-I5264.
18. Yamamoto Y, Zhao X, Suzuki AC, Takahasi S: Cysteine proteinase from the eggs of the silkworm, Bombyx mori : site of synthesis and a suggested role in yolk protein degradation. J Insect Physiol 1994, 40:447-454.

19. Homma K, Natori S: Identification of substrate proteins for cathepsin $L$ that are selectively hydrolyzed during the differentiation of imaginal disc of Sarcophaga peregrina. Eur J Biochem 1996, 240:443-447.

20. Zhao XF, Wang JX, Wang YC: Purification and characterization of a cysteine proteinase from eggs of the cotton boll worm, Helicoverpa armigera. Insect Biochem Mol Biol 1998, 28:259-264.

21. Cho WL, Tsao SM, Hays AR, Walter R, Chen JS, Snigirevskaya ES Raikhel AS: Mosquito cathepsin B-like proteinase involved in embryonic degradation of vitellin is produced as a latent extraovarian precursor. J Biol Chem 1999, 274:|33||-|332I.

22. $\mathrm{Xu}$ YS, Kawasaki $\mathrm{H}$ : Isolation and expression of cathepsin $\mathbf{B}$ cDNA in hemocytes during metamorphosis of Bombyx mori. Comp Biochem Physiol B 200I, I 30:393-399.

23. Fialho E, Nakamura A, Juliano L, Masuda H, Silva-Neto MAC: Cathepsin D-mediated yolk protein degradation is blocked by acid phosphatase inhibitors. Arch Biochem Biophys 2005, 436:246-253.

24. Shewale JG, Tang J: Amino acid sequence of porcine spleen cathepsin D. Proc Natl Acad Sci USA 1984, 81:3703-3707.

25. Boldbaatar D, Sikasunge CS, Battsetseg B, Xuan X, Fujisaki K: Molecular cloning and functional characterization of an aspartic protease from the hard tick Haemaphysalis longicornis. Insect Biochem Mol Biol 2006, 36:25-36.

26. Cho WL, Dhadialla TS, Raikhel AS: Purification and characterization of a lysosomal aspartic protease with cathepsin $D$ activity from mosquito. Insect Biochem 1991, 2 I: I65-I76.

27. Sakurai S, Kaya M, Satake S: Hemolymph ecdysteroid titer and ecdysteroid-dependent developmental events in the last larval stadium of the silkworm, Bombyx mori : role of low ecdysteroid titer in larval-pupal metamorphosis and a reappraisal of the head critical period. J Insect Physiol 1998, 44:867-88I

28. Gu SH, Chow YS: Analysis of ecdysteroidogenic activity of the prothoracic glands during the last larval instar of the silkworm, Bombyx mori. Arch Insect Biochem Physiol 2005, 58: I7-26.

29. Chinzei $Y$ : Induction of histolysis by ecdysone in vitro : Degradation of anterior silk gland in silkworm, Bombyx mori (Lepidoptera: Bombycidae). Appl Entomol Zool 1975, I0:136-138.

30. Sakurai S, Obara Y, Yasuhara N, Iwami M: Ecdysone, a substance that integrates the developmental changes of various tissues including morphogenesis, programmed cell death and energy metabolism at the pupal metamorphosis in insects. In Molecular Mechanisms of Insect Metamorphosis and Diapause Edited by: Suzuki A, Kataoka H, Matsumoto S. Tokyo: Industrial Publishing and Consulting; 1995:259-268.

31. Tsuzuki S, Iwami M, Sakurai S: Ecdysteroid-inducible genes in the programmed cell death during insect metamorphosis. Insect Biochem Mol Biol 200I, 31:32I-33I.

32. Park EJ, Burand JP, Yin CM: The effect of baculovirus infection on ecdysteroid titer in gypsy moth larvae (Lymantria dispar). J Insect Physiol 1993, 39:791-796.

33. Park EJ, Yin CM, Burand JP: Baculovirus replication alters hormone-regulated host development. I Gen Virol 1996, 77:547-554.

34. Clem RJ, Fechhelmer M, Miller LK: Prevention of apoptosis by a baculovirus gene during infection of insect cells. Science 1991, 254: $1388-1390$

35. Iwanaga M, Kang WK, Kobayashi M, Maeda S: Baculovirus infection blocks the progression of fat body degradation during metamorphosis in Bombyx mori. Arch Virol 2000, I45: I763-I77I.

36. Dean RL, Locke M, Collins JV: Structure of the fat body. In Comparative Insect Physiology, Biochemistry and Pharmacology Edited by: Kerkurt GA, Gilbert LJ. Oxford: Pergamon Press; 1985:I55-209.

37. Dittmer NT, Raikhel AS: Analysis of the mosquito lysosomal aspartic protease gene: an insect housekeeping gene with fat body-enhanced expression. Insect Biochem Mol Biol 1997, 27:323-335.

38. Ijiro T, Urakawa H, Yasukochi $Y$, Takeda M, Fujiwara Y: cDNA cloning, gene structure, and expression of Broad-Complex (BRC) genes in the silkworm, Bombyx mori. Insect Biochem Mol Biol 2004, 34:963-969. 
39. Nishita Y, Takiya S: Structure and expression of the gene encoding a Broad-Complex homolog in the silkworm, Bombyx mori. Gene 2004, 339:161-172.

40. Reza AMS, Kanamori Y, Shinoda T, Shimura S, Mita K, Nakahara Y, Kiuchi M, Kamimura M: Hormonal control of a metamorphosisspecific transcriptional factor Broad-Complex in silkworm. Comp Biochem Physiol B 2004, 139:753-76I.

4I. Kim SR, Lee KS, Kim I, Kang SW, Nho SK, Sohn HD, Jin BR: Molecular cloning of a cDNA encoding putative calreticulin from the silkworm, Bombyx mori. Int J Indust Entomol 2003, 6:93-97.

42. NCBI [http://www.ncbi.nlm.nih.gov/BLAST]

43. Je YH, Chang JH, Choi JY, Roh JY, Jin BR, O'Reilly DR, Kang SK: A defective viral genome maintained in Escherichia coli for the generation of baculovirus expression vectors. Biotechnol Lett 200I, 23:575-582

44. Lee KS, Kim SR, Park NS, Kim I, Kang PD, Sohn BH, Choi KH, Kang SW, Je YH, Lee SM, Sohn HD, Jin BR: Characterization of a silkworm thioredoxin peroxidase that is induced by external temperature stimulus and viral infection. Insect Biochem $\mathrm{Mol}$ Biol 2005, 35:73-84.

45. Wei YD, Lee KS, Gui ZZ, Yoon HJ, Kim I, Je YH, Lee SM, Zhang G.Z, Guo X, Sohn HD, Jin BR: N-linked glycosylation of a beetle (Apriona germari) cellulase Ag-EGase II is necessary for enzymatic activity. Insect Biochem Mol Biol 2006, 36:435-44I.

46. Hong HK, Woo SD, Choi JY, Lee HK, Kim MH, Je YH, Kang SK: Characterization of four isolates of Bombyx mori nucleopolyhedrovirus. Arch Virol 2000, I45:235I-236I.

Publish with Bio Med Central and every scientist can read your work free of charge

"BioMed Central will be the most significant development for disseminating the results of biomedical research in our lifetime. "

Sir Paul Nurse, Cancer Research UK

Your research papers will be:

- available free of charge to the entire biomedical community

- peer reviewed and published immediately upon acceptance

- cited in PubMed and archived on PubMed Central

- yours - you keep the copyright 